Open Access

\title{
Prognostic factors for surgical outcome in spinal cord injury associated with ossification of the posterior longitudinal ligament (OPLL)
}

Soon Young Kwon ${ }^{1}$, Jun Jae Shin ${ }^{1 *}$, Ji Hae Lee ${ }^{2}$ and Woo Ho Cho ${ }^{2}$

\begin{abstract}
Background: Ossification of the posterior longitudinal ligament (OPLL) may increase the risk of spinal cord injury (SCl) with various neurological deficits after minor trauma. However, few studies have investigated the influence of OPLL on neurological outcome after acute cord injury. We examined whether severe spinal canal stenosis caused by OPLL affects neurological outcome after SCI based on intramedullary signal intensity (SI) changes on magnetic resonance imaging (MRI).

Methods: From June 2006 to July 2013, we treated 246 patients with cervical cord injury. Fifty-one (20.7 \%) patients had ventral cord compression due to OPLL without any bony fractures. Among them, 38 patients (34 men, mean age 62.7 years) underwent cervical laminoplasty (8) and cervical decompression and fixation (30). The neurologic assessments were performed in patients who had 1-year follow-up, and the mean follow-up period was 42.2 months. OPLL type, cause of injury, cervical sagittal angle, cervical spine stenosis, cord compression ratio (space available for the spinal cord (SAC)), and grade of intramedullary SI (grade 0, none; grade 1, light; grade 2, intense T2WI) were assessed.

Results: Mean American Spinal Injury Association (ASIA) motor score at admission was $38.4 \pm 21.9$ (range, 2-70) and improved to $67.7 \pm 19.1$ (range, 8-94) at last follow-up ( $p<0.05$ ). Mean recovery rate of the motor score was $55.8 \pm$ $19.9 \%$. Five patients had SI grade 0, 20 patients had SI grade 1, and 13 patients had SI grade 2. Among the variables tested, age, initial ASIA motor grade, intramedullary SI grade, and SAC were significantly related to neurological outcome. However, initial cervical alignment, canal diameter, length of SI, time interval between injury and operation, and OPLL type had no significant effect on neurological outcome.

Conclusions: Preoperative neurological status, cord compression ratio, and SI grade are related to neurological outcome in patients with SCl associated with OPLL. The better the preoperative neurological status, the more favorable the neurological outcome after surgery. A higher SI grade on preoperative T2WI was negatively related to neurological outcome. Therefore, the severity of SI change, cord compression ratio, and preoperative neurological status can be regarded as significant prognostic factors in patients with SCl associated with OPLL.
\end{abstract}

Keywords: Ossification of the posterior longitudinal ligament, Spinal cord injury, Magnetic resonance imaging

\footnotetext{
* Correspondence: zunzae@hanmail.net

${ }^{1}$ Department of Neurosurgery, Sanggye Paik Hospital, Inje University College of Medicine, Sanggye-7 dong, 761-1, Nowon-gu, Seoul 139-707, South Korea Full list of author information is available at the end of the article
} 


\section{Introduction}

Ossification of the posterior longitudinal ligament (OPLL) is a common cervical disease that can cause stenotic changes in the spinal canal and contribute to the development of quadriparesis. OPLL has been found in about $30 \%$ of patients with spinal cord injury (SCI) $[1,2]$. These results indicate that OPLL is a major risk factor for developing acute cervical SCI. Several factors for predicting surgical outcome after surgical treatment of cervical OPLL have been discussed [3-6]. Some authors reported that patients with increased intramedullary signal intensity (SI) based on magnetic resonance imaging (MRI) had a poor prognosis after surgical decompression, while others found no clear relationship between SI and prognosis after surgery [3, 5-9]. Only a handful of studies have investigated prognostic factors for SCI associated with OPLL $[2,10,11]$. It is difficult to predict the recovery rate and prognosis of SCI with OPLL because traumatic cervical SCI patients are a heterogeneous patient population and are treated with various management methods.

We performed a retrospective investigation to examine prognostic factors in SCI associated with OPLL without any bony fracture. We assessed whether severe spinal canal stenosis caused by OPLL, intramedullary signal change on magnetic resonance images, or other potential prognostic factors affected neurological outcome after SCI without any bony fractures.

\section{Materials and methods \\ Patient selection}

This study was approved by the local ethical committee and the institutional review board of our hospital. From June 2006 to July 2013, we treated 246 patients with cervical cord injury. Among the patients treated, 51 patients (20.7\%) had ventral cord compression involving more than three-level OPLL without bony fractures. Thirteen patients who underwent anterior cervical decompression were excluded because of bias or differences in surgical factors.

Thirty-eight patients (34 men) with a mean age of 62.7 years (range, 40 to 82 years) had spinal cord injury without any bony fractures associated with more than three-level OPLL. All patients underwent posterior cervical decompressive surgery using cervical laminoplasty or laminectomy with fusion.

Patient age, gender, type of trauma, duration of symptoms, neurologic deficits, medical conditions, and radiographic findings were investigated. Charts were reviewed to determine the cause of injury and the interval between the accident and operation. Force was confirmed by edema of prevertebral soft tissue on MRI or frontal ecchymosis. Presence of OPLL was confirmed by X-ray or computed tomography.
Inclusion criteria were cervical cord injury and admission through the outpatient department (OPD) or emergency room (ER). Patients with spinal cord compression caused by OPLL extending more than three vertebrae were included, as were those with the specific incomplete neurological syndrome called central cord syndrome, which is characterized by greater muscle weakness and/or sensory loss in the upper limbs than the lower limbs and others caused by hyperextension, hyperflexion, or axial loading injuries.

We excluded patients with neurological symptoms before trauma, one- or two-level OPLL, other acute traumatic cord injuries with cervical bony fracture or dislocation caused by high-energy trauma, complete neurological injury after trauma, or another confirmed neurological disorder (e.g., cerebral palsy, Parkinson's disease, multiple sclerosis, polio). Ossification of the yellow ligament, severe disc herniation, and previous history of cervical spine surgery were also exclusion criteria.

All patients underwent high-resolution MR imaging using a $1.5 \mathrm{~T}$ Signa (Siemens Medical System) imaging unit prior to surgery. We carefully ruled out tumors, demyelinating disease, sarcoid lesions, and spinal cord infarct. Patients who died or had missing data before follow-up evaluation were excluded.

We defined complications as events that occurred within 1 month after surgery. We analyzed neurologic recovery in patients who had completed at least 12 months of follow-up.

\section{Neurological evaluation}

Neurological status was assessed using the International Standards for Neurological and Functional Classification of Spinal Cord Injury according to the American Spinal Injury Association (ASIA). ASIA impairment scale and motor score (ranging from 0 to 100) were documented at the time of admission and discharge for each patient, and at about 12 months after surgery. The neurological assessments were performed in patients who had completed 1-year follow-up (5 patients underwent 12-month follow-up postoperatively and 33 patients 24-month follow-up after surgery).

Improvement in neurologic score was determined by calculating the recovery rate using the method of Lucas and Ducker [12]. Recovery rate (RR) takes into account the impact of changes dependent on baseline values by implying the largest potential improvement: $\mathrm{RR}=\left(\mathrm{NS}_{\mathrm{f}}-\mathrm{NS}_{\mathrm{i}}\right) /\left(T-\mathrm{NS}_{\mathrm{i}}\right)$, where RR is the recovery rate, $\mathrm{NS}_{\mathrm{f}}$ is the neurologic score at follow-up, $\mathrm{NS}_{\mathrm{i}}$ is the initial neurologic score, and $T$ is the maximum possible score $(100,114$, and 114 for the motor score, sensory score, and pin-prick score, respectively). 


\section{Radiological evaluation}

Preoperative plain radiographs, three-dimensional computed tomography (3D CT), and MRI of the cervical spine were performed in all patients. Radiographic parameters include the type of OPLL, sagittal diameter of the canal, cervical lordosis, space available for the spinal cord (SAC), and intramedullary signal intensity on T2weighted MR images were recorded. OPLL was classified as segmental type, continuous type, mixed type, or localized type according to the criteria of the Japanese Investigation Committee on the Ossification of the Spinal Ligament [13].

Cervical spine stenosis was measured by the TorgPavlov ratio (ratio of the sagittal spinal canal diameter to the vertebral body diameter at the same level) on plain radiographs. Cervical sagittal alignment (lordotic angle) was determined according to Cobb's method by drawing a line parallel to the inferior aspect of the $\mathrm{C} 2$ body and a line parallel to that of the $\mathrm{C} 7$ body from a neutral lateral view. Also, the K-line slope [14], the straight line of the midpoints from $\mathrm{C} 2$ to $\mathrm{C} 7$ spinal canal, was used as a radiological parameter (Fig. 1).

A computed tomographic (CT) scan was performed before surgery for all patients. An Aquilion 16 CT scanner (Toshiba Medical Systems, Tokyo, Japan) was used in this series. Horizontal slices were cut to a slice thickness of $1 \mathrm{~mm}$ in all cases. Three-dimensional CT was performed at a neutral cervical position to avoid neurological deterioration in cervical injured patients with cervical OPLL. Using axial CT scans, we evaluated static canal stenosis caused by OPLL by measuring the SAC (Fig. 2).

Using a picture archiving and communication system (PACS), we measured the length of the signal change from one end of the involved region to the other on T2weighted sagittal images (Fig. 3).

We divided the signal intensity (SI) on T2-weighted MR images into three grades. Increased SI of the spinal cord at the narrowest level was assigned one of three grades (grades 0,1 , or 2) by experienced radiologists. Observers were blinded to the patient data. SI was classified as grade 0 if there was no intramedullary high SI on T2-weighted MR images, grade 1 if a predominantly faint and fuzzy border was noted, and grade 2 if a predominantly intense and well-defined border was noted (Fig. 4).

\section{Surgical management}

We attempted to perform cervical decompressive surgeries in patients with neurological deficits as soon as possible. If the spinal cord was injured within $8 \mathrm{~h}$, patients with neurological deficit after minor trauma received a high dose of prednisolone, according to the American National Acute Spinal Cord Injury Society criteria [15]. The first dose was $30 \mathrm{mg} / \mathrm{kg}$ and given within $15 \mathrm{~min}$; after $45 \mathrm{~min}$, a second dose of $5.4 \mathrm{mg}$ $(\mathrm{kg} / \mathrm{h})$ was maintained for $23 \mathrm{~h}$. If the injury time exceeded 8 h, $20 \mathrm{mg}$ dexamethasone was used four times a day for the first 3 days and daily reduced for the next 3 days. The decision to perform decompressive surgery was based on the patient's general and neurological status. We defined the operation time as the time interval between the traumatic event and surgery.

Cervical extensive laminoplasty was selected based on the neurologic symptoms and the extent of cord compressed levels, including the most narrowed side on images. Unilateral or bilateral gutters were made using a high-speed drill at the area between the facet joints and the laminae, and spinous processes were split sagittally with a high-speed burr. The spinal canal was enlarged by opening the split laminae unilaterally or bilaterally with a spreader. Posterior cervical titanium miniplates (Centerpiece ${ }^{\oplus}$, Medtronic Sofamor Danek, Memphis, TN, USA) or hydroxyapatite spacers (Apacerum, Asahi Optical Co., Ltd., Tokyo, Japan) were used to keep the "door" open.

For OPLL patients with local kyphosis or segmental instability, we performed cervical laminectomy and fusion and tried to realign local kyphosis using a posterior screw-rod system. Following complete decompression of neural elements, lateral mass screw and rod instrumentation were performed. Perizygapophysial joints were filled with autologous bone fragments from the excised lamina. After surgery, all patients were required to wear Miami neck collars or Philadelphia braces for a mean period of 3 months. Patients were followed clinically by radiography using plain and dynamic films at 1,3 , and 6 months and then every 6 months postoperatively.

\section{Statistical analysis}

Multifactorial effects of variables were studied. MannWhitney $U$ tests were used for nonparametric analyses of differences between pairs of groups, and the KruskalWallis test followed by the Mann-Whitney $U$ test was used to examine differences between the three SI groups. To identify variables independently related to ASIA recovery ratio, univariate or multivariate logistic regression analyses were used. Pearson's correlation analysis was performed to determine the significance of the correlation between ASIA recovery ratio and age, duration of symptoms, cervical alignment, cervical canal stenosis (Pavlov's ratio), SAC, preoperative ASIA motor score, SI length, and postoperative ASIA motor score. Spearman's correlation analysis was performed to determine the significance of the correlation between ASIA recovery ratio and gender, OPLL type, or preoperative SI grade. Correlations between variables were assessed with regression analyses using MedCalc version 14.0 (MedCalc, 


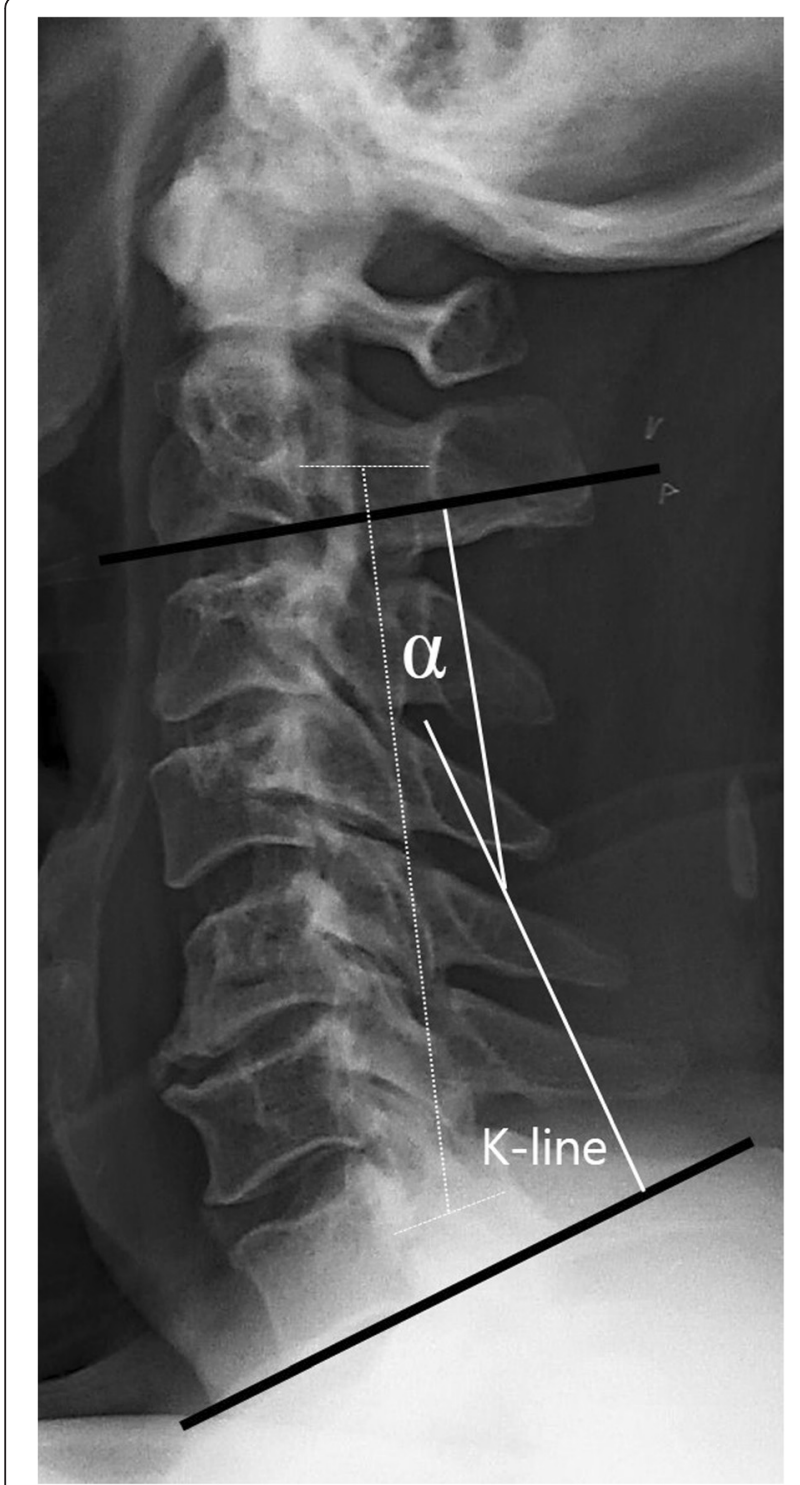

Fig. 1 Cervical lordosis and K-line. Lordotic angle was created by drawing a line parallel to the inferior aspect of the C2 body and a line parallel to that of the $\mathrm{C} 7$ body on lateral view with the patient in a neutral position. The $K$-line is a straight line from the midpoints of the spinal canal at C2 and C7

Mariakerke, Belgium). $p$ values $<0.05$ were considered significant.

\section{Results}

The primary cause of cord injury in patients with OPLL was slipping down and falling injuries (78.9\%), followed by a motor vehicle accident. The patient had episodes of minor neck trauma: injuries from slipping down in 23, falling down in 7 patients, motor vehicle accident in 7 , being struck by objects in 1, and sports activities in 2 . The injury was mostly caused by a sudden force with subsequent compression of the cord by a bony posterior

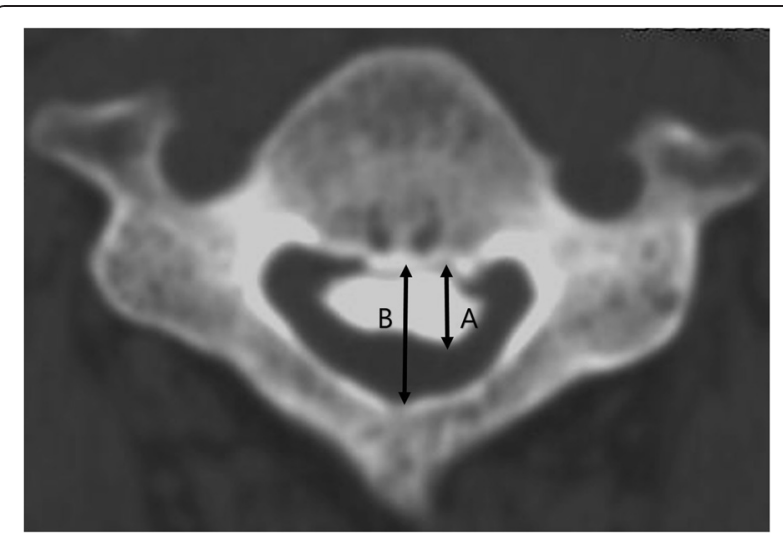

Fig. 2 Measurement of the space available for the spinal cord (SAC). SAC was calculated on the basis of axial computed tomography. $B=$ developmental canal diameter. $B-A=$ space available for the spinal cord (SAC)

ligament or narrowed canal. Most cases of the cervical SCI complicated by OPLL are caused by hyperextension injuries resulting from falling or slipping down injuries. Fourteen patients had typical central cord syndrome. Four had incomplete cord injuries caused by flexion injuries, and 20 had incomplete neurological deficits by combined injuries such as lateral bending, rotation, axial loading, and hyperflexion or hyperextension of the cord. All 38 patients included in this study underwent posterior decompressive surgery. Expansive cervical laminoplasty was performed in 8 patients, and cervical laminectomy with fusion was performed in 30 patients.

Mean ASIA motor score at admission was $38.4 \pm 21.9$ (range, 2-70) and improved to $67.7 \pm 19.1$ at last followup (range, 8-94) $(p<0.05)$. Overall, the mean recovery rate of the motor score was $55.8 \pm 19.9 \%$ (range, 8.2$92.8 \%$ ), and the recovery ratio improved in all patients. In addition, the average ASIA score of light touch sensation and pin-prick sensation increased from $70.9 \pm 34.5$ and $70.9 \pm 34.4$, respectively, at admission to $79.7 \pm 34.1$ and $79.7 \pm 34.1$, respectively, after the final follow-up. Mean recovery rate of light touch and pin sensation were $32.3 \pm$ $28.2 \%$ and $33.8 \pm 24.7 \%$, respectively. In general, the sensory recovery ratio was lower than the motor recovery ratio. Mean follow-up time was $42.2 \pm 25.1$ months (range, 16.8-97.8 months). The duration of preoperative symptoms ranged from 1 to 23 days. SAC was $6.1 \pm 2.2 \mathrm{~mm}$ (range, 2.6-10.6 mm). The mean length of SI changes was $5.9 \pm 3.7 \mathrm{~mm}$ (range, $0-13.9 \mathrm{~mm}$ ).

Radiological findings revealed continuous in 14 patients, mixed type OPLL in 4 patients, and segmental type OPLL in 20 patients. There was no significant relationship between OPLL type and neurological outcome in our study. The mean OPLL level treated by surgical operation was $3.2 \pm 0.4$. Among 38 patients, 33 (86.8\%) showed increased signal intensity within the spinal cord 


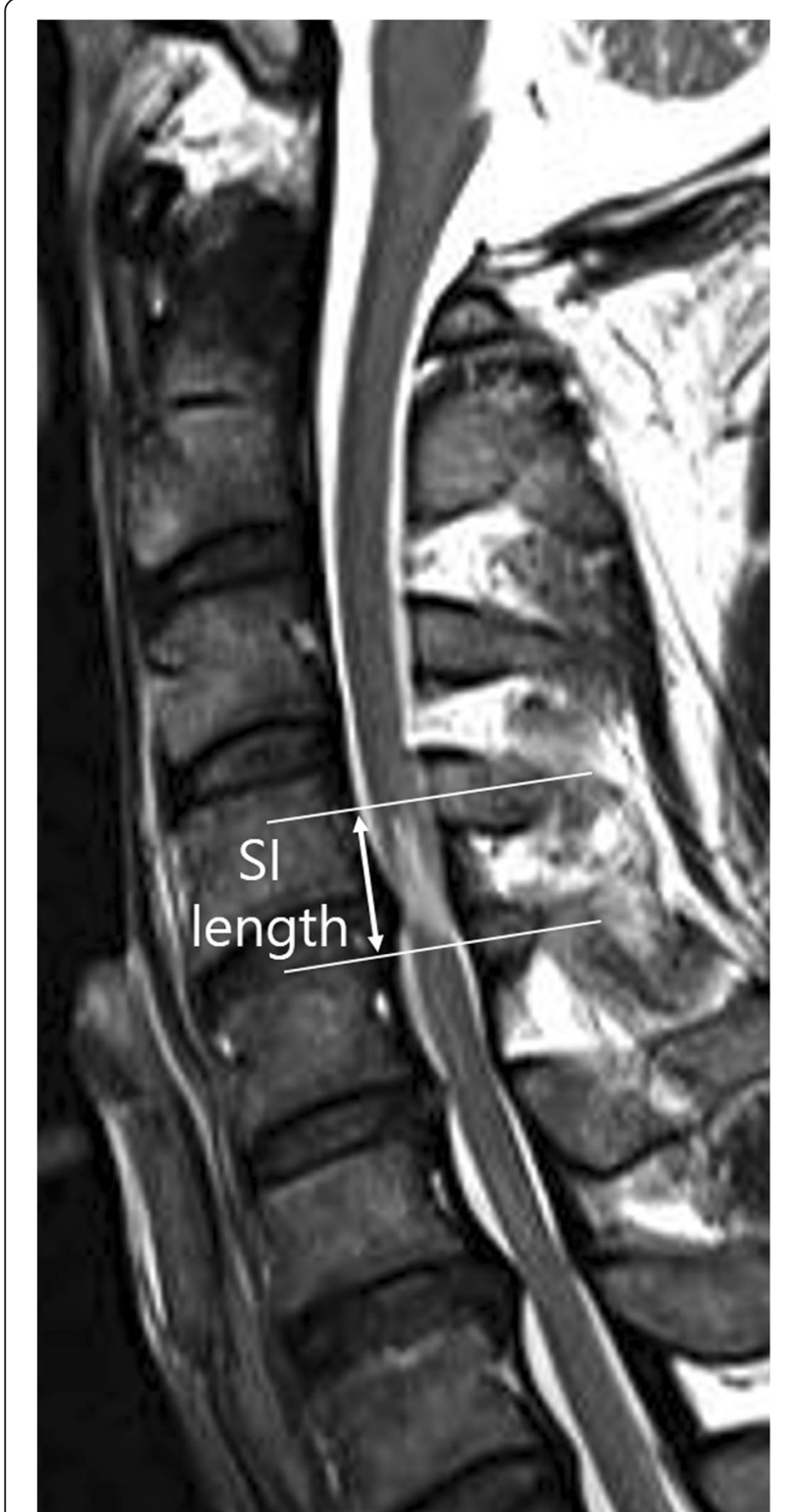

Fig. 3 Length of signal intensity. It was measured by the proximaldistal range of the intramedullary hyperintensity on T2-weighted images

on T2-weighted MR images, whereas 5 patients (13.2\%) did not. SI change on MR images was located on $\mathrm{C} 3 / 4$ level in 13 patients, $\mathrm{C} 4 / 5$ in $14, \mathrm{C} 5 / 6$ in $4, \mathrm{C} 2$ / $3 / 4$ in 1 and $C 3 / 4 / 5$ in 1 . The mean length of SI changes was $5.9 \pm 3.7 \mathrm{~mm}$ (range, 0-13.9 mm). However, the length of pathological changes on spinal cord was not correlated with neurological outcome. When the grade of intramedullary SI change on T2WI was assessed, 5 patients showed SI grade 0 changes, 20 patients SI grade 1 changes, and 13 patients grade 2 changes. In this study, there was no low-signal change on T1-weighted image initially.
The respective surgical level caused by OPLL was $3.4 \pm$ 0.5 in grade 0 patients, $3.1 \pm 0.3$ in grade 1 , and $3.2 \pm 0.4$ in grade 2 . There was no significant difference in surgical levels among the three groups. The surgical procedures performed were expansive laminoplasty in one patient with grade 0 and cervical laminectomy with fusion in four patients with grade 0 . In grade 1 , expansive laminoplasty was performed in 4 patients and cervical laminectomy with fusion in 16 . In grade 2, expansive laminoplasty was performed in three patients and cervical laminectomy with fusion in ten (Table 1).

Preoperative motor scores and recovery ratios (\%) were $60.0 \pm 10.1$ and $82.4 \pm 18.1 \%$, respectively, in the five SI grade 0 patients; $40.6 \pm 21.1$ and $66.0 \pm 18.1 \%$, respectively, in the 20 grade 1 patients; and $26.0 \pm 6.9$ and $30.9 \pm 26.6 \%$, respectively, in the 13 grade 2 patients. Preoperative sensory (touch) scores and recovery ratios (\%) were $100.8 \pm 7.6$ and $69.8 \pm 19.7 \%$, respectively, in the five grade 0 SI patients; $77.5 \pm 29.2$ and $29.7 \pm 19.2 \%$, respectively, in the 20 grade 1 patients; and $49.4 \pm 36.7$ and $21.8 \pm 22.2 \%$, respectively, in the 13 grade 2 patients. The motor and sensory recovery ratios were significantly different among the three groups $(p<0.05)$ (Table 1). That is, patients with a higher grade of intramedullary signal intensity showed poorer ASIA motor and sensory recovery ratio than those with a lower grade of signal changes on T2-weighted images (Fig. 5).

In present study, 29 patients were classified as K-line $(+)$ and 9 were classified as K-line $(-)$. The mean recovery ratio was $63.7 \pm 24.3 \%$ in the K-line $(+)$ group and $30.3 \pm 25.6 \%$ in the K-line (-) group. The neurological outcome in patients with K-line $(+)$ was better than in those with K-line $(-)(p<0.05)$.

\section{Logistic regression analyses}

Univariate logistic regression analysis and multivariate logistic regression analysis were performed to identify prognostic factors. Patient age, initial ASIA motor score, severity of intramedullary signal intensity, and SAC had significant effects on the neurological outcome after surgery (Table 2). Patient age was significantly correlated with recovery ratio (coefficient $=-1.0733,95 \% \mathrm{CI}=-1.8715--$ $0.2752, p=0.0098$, Fig. 6a). Initial ASIA motor score was significantly correlated with recovery ratio (coefficient = 0.7533, $95 \%$ CI $=0.5711-0.8647, p<0.0001$, Fig. 6b). Furthermore, the change in SAC was significantly correlated with the rate of postoperative neurologic improvement (coefficient $=0.3967,95 \% \mathrm{CI}=0.07842-0.6416, p=0.0166$, Fig. 6c). However, there was no significant relationship between initial cervical curvature, time interval between injury and operation, OPLL type, the length of signal intensity (SI), or congenital canal stenosis (Pavlov's ratio) and neurological outcome. 


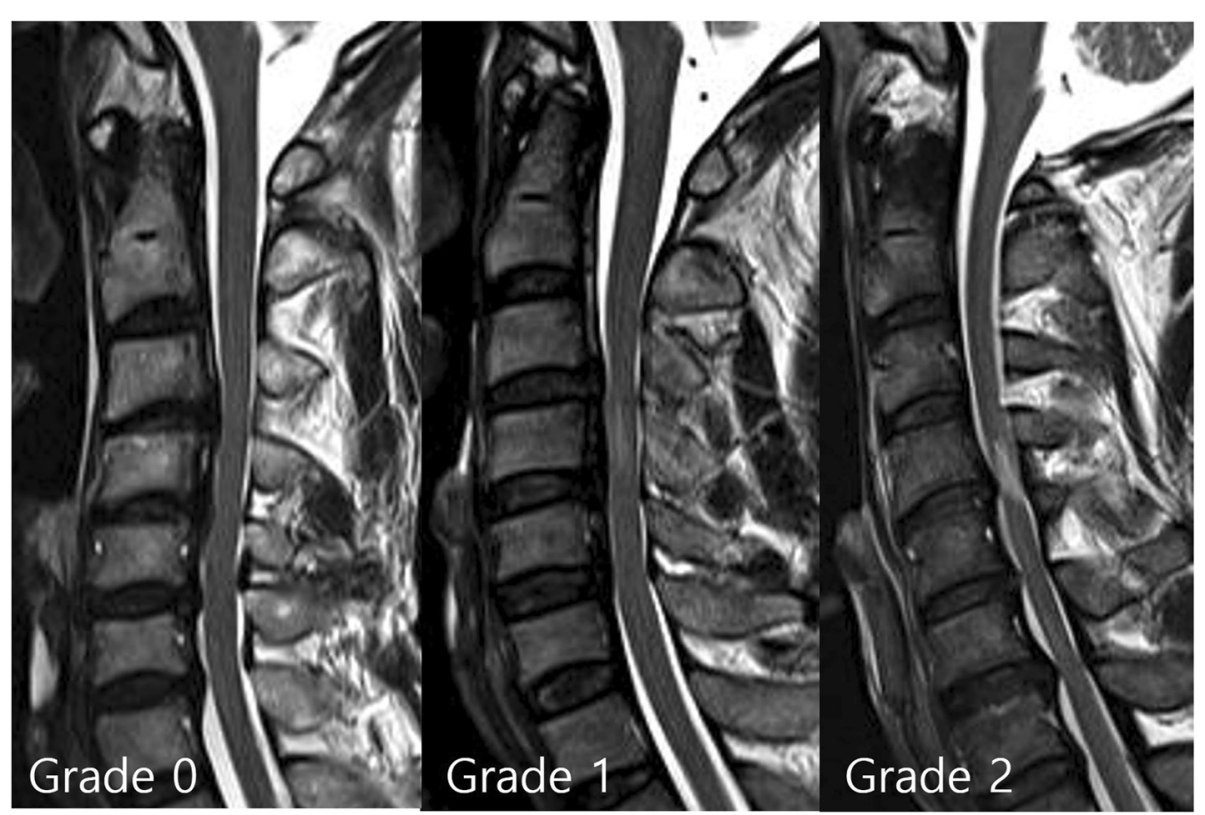

Fig. 4 Signal intensity grade. Signal intensity of the spinal cord was assessed as grade 0_ no change in signal intensity (SI) on T2-weighted MR images; grade 1-light intensity change; and grade 2-bright signal, clearly distinguishable from that of grade 1

In our study, 1 of the 38 patients (2.6\%) developed C5 palsy and postoperative hematoma $3 \mathrm{~h}$ after surgery. We immediately removed the hematoma, but the C5 nerve palsy remained on the patient's right side. Another patient developed superficial wound infection after surgery, but no additional surgery was required to remove instrumentation.

\section{Discussion}

OPLL has been found to be highly prevalent in patients with acute traumatic cervical SCI; in particular, OPLL has been reported in $34 \sim 38 \%$ of cervical SCI patients without bone injury $[1,2]$. They were caused primarily by low-energy trauma. The vast majority of patients were elderly and unaware of OPLL. According to other studies [16], there are only a few patients who are aware of having cervical OPLL before trauma. In our cases, one patient suffered neck pain 2 years before the trauma but he was not aware of cervical OPLL and was not diagnosed with cervical OPLL. Consistent with previous studies, the majority of SCI patients with OPLL in our study were elderly, with the peak incidence found in patients between 65 and 75 years old of age.

Intramedullary signal changes and neurological outcome Several studies have investigated the relationship between increased SI on MRI and surgical outcomes in

Table 1 Clinical characteristics and neurological outcome according to the severity of signal intensity

\begin{tabular}{lllll}
\hline & Grade $0(n=5)$ & Grade $1(n=20)$ & Grade $2(n=13)$ & $p$ value \\
\hline Age (years) & $57.4 \pm 6.1$ & $63.8 \pm 11.5$ & $63.2 \pm 11.4$ & $0.523 \mathrm{NS}$ \\
Symptom duration (days) & $3.8 \pm 4.4$ & $3.8 \pm 4.9$ & $2.2 \pm 1.7$ & $0.634 \mathrm{NS}$ \\
SAC (mm) & $7.2 \pm 1.5$ & $6.6 \pm 2.4$ & $16 \pm 1.6$ & $0.056 \mathrm{NS}$ \\
Cervical curvature ( $\left.{ }^{\circ}\right)$ & $13.3 \pm 4.1$ & $15.1 \pm 6.9$ & $0.8 \pm 9.8$ & $0.769 \mathrm{NS}$ \\
Cervical canal stenosis & $0.84 \pm 0.27$ & $0.69 \pm 0.17$ & $26.0 \pm 6.9$ & $0.174 \mathrm{NS}$ \\
Preoperative motor score & $60.0 \pm 10.1$ & $40.6 \pm 21.1$ & $49.4 \pm 36.7$ & 0.033 \\
Preoperative touch score & $100.8 \pm 7.6$ & $77.5 \pm 29.2$ & $30.9 \pm 26.6$ & 0.003 \\
Recovery motor rate (\%) & $82.4 \pm 18.1$ & $66.0 \pm 18.1$ & $21.8 \pm 22.2$ & 0.0005 \\
Recovery touch rate (\%) & $69.8 \pm 19.7$ & $29.7 \pm 19.2$ & $3.2 \pm 0.4$ & 0.0009 \\
Surgical levels & $3.4 \pm 0.5$ & $3.1 \pm 0.3$ & 10 & $0.267 \mathrm{NS}$ \\
Laminectomy and fusion & 4 & 16 & 3 & \\
Expansive laminoplasty & 1 & 4 & & \\
\hline
\end{tabular}

SAC space available for the spinal cord, NS not significant 


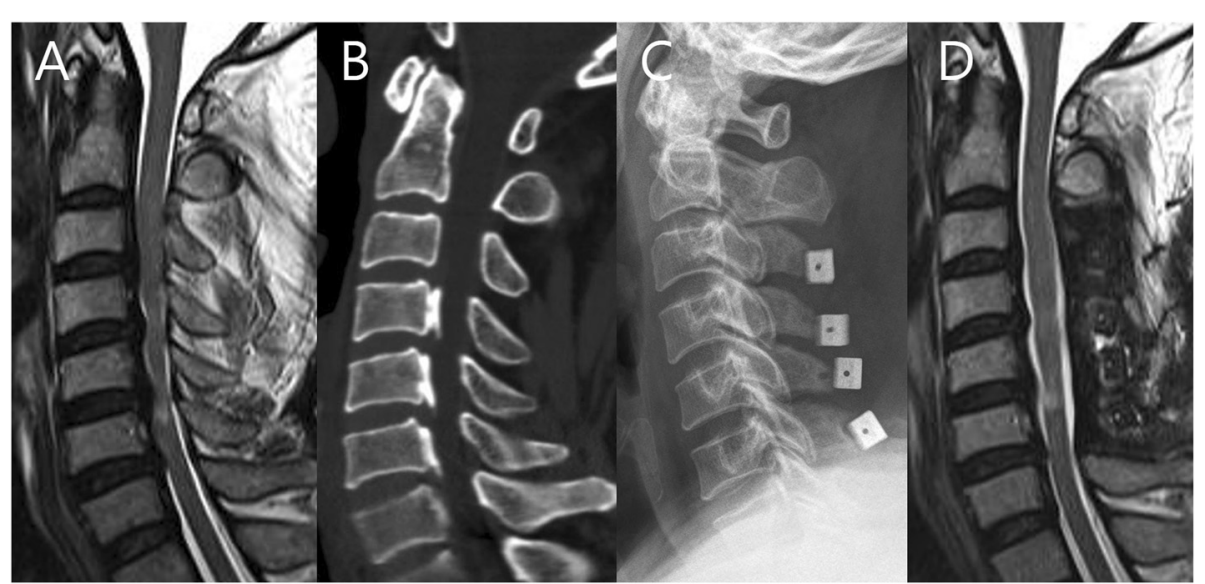

Fig. 5 A patient with intramedullary signal intensity grade 2. a Preoperative T2-weighted MRI results for a 54-year-old male patient with spinal cord compression at C3-4-5-6-7. Intramedullary high signal intensity (SI), grade 2, was visible at the region of the marked compressed cord. Initial ASIA motor score was 48. b Cervical 3D CT showed mixed type ossification of the posterior longitudinal ligament (OPLL). Spinal canal stenosis (SAC) was $5.9 \mathrm{~mm}$. c The patient underwent cervical laminoplasty at C3-4-5-6. d MRI of the patient from B 12 months after cervical laminoplasty with successful decompression of the spinal cord. The patient's ASIA motor score was 72 . The recovery rate was $46.2 \%$

patients with acute cervical cord injury $[3,5,6,17,18]$. We found that if intramedullary signal changes on T2WI were present, clinical outcomes were unfavorable after surgery as compared to those patients with no intramedullary signal changes on T2WI. We recognized three grades of cord SI and found that these grades were inversely proportional to the outcome of cervical cord injury. Patients with a brighter SI change (grade 2) on T2-weighted MR images had poorer neurological outcomes than those with a faint SI change (grade 1) or those without a SI change (grade 0). Thus, decompressive surgery should be performed for patients with high intramedullary SI if possible.

Ramanauskas and colleagues [19] divided myelomalacia into three stages and speculated that in the early stage, a change in the signal intensity of the spinal cord on MRI reflects cord edema. In contrast, in the intermediate stage, a signal change reflected cystic necrosis of the central gray matter after prolonged cord edema. They reported that in early and intermediate stages, the spinal cord showed high-signal intensity on T2WI sequences, while at a later stage, low-signal intensity on T1WI and high SI on T2WI sequences were noted. We did not find a low-signal change on T1-weighted images initially. We assumed that this was because patients experienced a sudden minor traumatic force to their neck area, and there was not sufficient time for low SI changes to develop on T1WI.

\section{Age and neurological outcome}

Age as an adverse prognostic factor for neurological outcome has been reported previously $[4,20]$. Prognosis was less optimistic in patients with severe initial cord injury who were older than those who were younger [20]. We also found that older age at injury was a negative predictor of neurological recovery. Older patients tend to have a higher incidence of cervical spondylosis and cord ischemia due to atherosclerotic changes of the vertebral or feeding vascular structures. These senile factors and other comorbidities might contribute to unfavorable clinical outcomes in elderly patients with an injured spinal cord and OPLL.

Table 2 Multivariate regression analysis results for the recovery ratio and other factors

\begin{tabular}{lccclc}
\hline Recovery ratio & Coef. & s.e. & t value & $p$ value & $95 \%$ Cl \\
\hline Age & -1.073 & 0.394 & -2.727 & 0.0098 & -1.872 to -0.275 \\
Operation interval & 0.737 & 1.151 & 0.640 & $0.526 \mathrm{NS}$ & -1.598 to 3.071 \\
Cervical alignment & 0.284 & 0.611 & 0.464 & $0.645 \mathrm{NS}$ & -0.956 to 1.523 \\
Cervical stenosis (Pavlov ratio) & 1.958 & 25.642 & 0.0763 & $0.939 \mathrm{NS}$ & -50.099 to 54.014 \\
SAC & 4.822 & 1.913 & 2.520 & 0.016 & 0.934 to 8.711 \\
SI length & -0.868 & 0.660 & -1.316 & $0.196 \mathrm{NS}$ & -2.207 to 0.470 \\
SI grade & -7.996 & 5.328 & -5.254 & $<0.001$ & -8.802 to -3.189 \\
Preoperative motor score & 0.876 & 0.128 & 6.872 & $<0.001$ & 0.618 to 1.135 \\
\hline
\end{tabular}

SAC space available for the spinal cord, SI signal intensity, Coef. regression coefficient, s.e. standard error, NS non-significant 

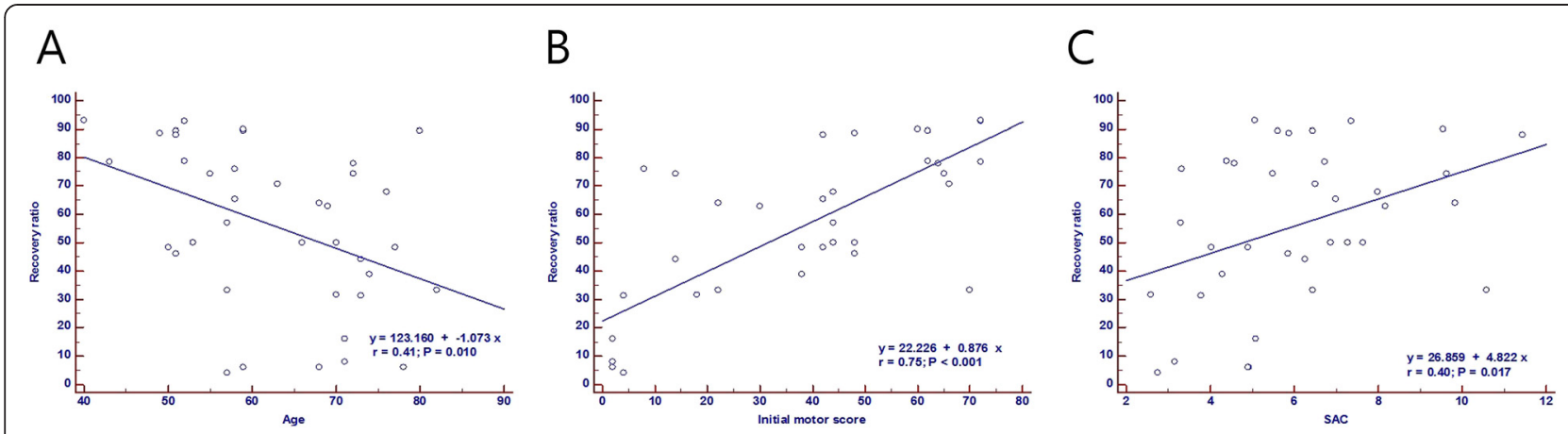

Fig. 6 The correlation between age, ASIA motor score, SAC, and recovery ratio. a The patients' age showed a significant correlation with the recovery ratio. $\mathbf{b}$ Initial ASIA motor score was correlated with recovery ratio. c Recovery ratio was correlated with the space available for the spinal cord (SAC)

\section{Canal stenosis and neurological outcome}

Minor trauma to the neck sometimes results in deterioration of symptoms in patients with cervical OPLL because of preexisting spinal canal narrowing by OPLL [21-23]. Previous investigators noted poor neurological outcome in spinal cord injured patients who had severe cord compression [24-26]. Furthermore, developmental cervical spinal canal stenosis may lead to transient compression of the spinal cord and affect the neurological outcome after surgery [23]. In the present study, we found that the extent of cord compression, as assessed by parameters such as SAC, was correlated with neurologic recovery in SCI patients with OPLL, but the effect of the developing cervical canal on neurological recovery was not significant. At the moment of traumatic injury, the spinal cord can be abruptly pinched by inner structures of the spinal canal, particularly in severe stenotic areas caused by OPLL. The traumatic force may cause intramedullary SI changes to develop, resulting in neurologic deficits. Lesions of intramedullary SI change were in areas adjacent to the severely compressed spinal cord. We found that the extent of cord compression was a more important predictor of neurological outcome after surgery than congenital canal stenosis (Pavlov's ratio) in cervical cord injured patients with OPLL.

\section{Initial neurological status and neurological outcome}

Initial ASIA motor score has been shown to be significantly related to ambulatory and hand function outcomes in patients with spinal cord injury [21, 25]. In agreement with other studies [21, 26-28], we found that preoperative neurological status, particularly motor score, predicted neurological recovery. One interesting finding of this study was that the ASIA sensory recovery ratio was lower than the ASIA motor recovery ratio. This suggests that if patients with minor traumatic injury have high intramedullary SI changes in the spinal cord, their sensory function improves less than their motor recovery.
Fujiyoshi and colleagues [14] reported the K-line as a simple and practical index for deciding the surgical approach for cervical OPLL patients and asserted the correlation of cervical alignment with neurological outcome. They advocated that the patients with a negative K-line slope, which meant the OPLL touched the spinal cord, had a significantly lower recovery rate than those with a positive K-line. In accordance with previous studies [14, 29, 30], our present study showed the better recovery rate in the patients with positive $\mathrm{K}$-line than in negative K-line.

Limitations of this study include its retrospective design and the small number of patients. The type of trauma may also have been more varied than we accounted for because it was patient-reported. Furthermore, the definition of a minor traumatic injury is controversial. Despite these drawbacks, our results provide insight into the factors that predict neurological recovery in patients with OPLL who undergo decompression surgery due to an injured cervical cord. We did not perform a quantitative analysis of signal intensity in the spinal cord. Rather, radiologists blinded to the patient data categorized intramedullary signal intensities into three groups. Further extended and comprehensive studies are required to confirm the specific relationships between prognostic factors and clinical outcomes described in this paper.

\section{Conclusions}

The degree of canal stenosis, including SAC, intramedullary signal intensity change, initial motor score, and patient age, should be considered prognostic factors when surgical decompression is performed for cervical cord injured patients without bony fractures, particularly for those patients who have a high intramedullary SI initially. That is, prognosis was less optimistic in older patients with severe neurologic deficits, marked cord compression, or the existence of abnormal MR signal change in the spinal cord. 


\section{Competing interests}

The authors declare that they have no competing interests.

\section{Authors' contributions}

SYK carried out the analysis of data and drafted the manuscript. JJS revised it critically for important intellectual content and gave final approval of the version to be submitted. JHL participated in the design of the study and performed the statistical analysis. WHC conceived of the study, and participated in its design and coordination and helped to draft the manuscript. All authors read and approved the final manuscript.

\section{Acknowledgements}

The authors wish to thank Hyun Kyung Park, Ph.D., for her contributions in drafting the manuscript or revising it for important intellectual content. The authors also extend gratitude to Ms. Ah Ram Lee for creating the original artwork for the figures and acquisition of data in this publication.

\section{Author details}

'Department of Neurosurgery, Sanggye Paik Hospital, Inje University College of Medicine, Sanggye-7 dong, 761-1, Nowon-gu, Seoul 139-707, South Korea. ${ }^{2}$ Department of Radiology, Sanggye Paik Hospital, Inje University College of Medicine, Sanggye-7 dong, 761-1, Nowon-gu, Seoul 139-707, South Korea.

Received: 17 March 2015 Accepted: 28 May 2015

Published online: 12 June 2015

\section{References}

1. Katoh S, el Masry WS, Jaffray D, McCall IW, Eisenstein SM, Pringle RG, et al. Neurologic outcome in conservatively treated patients with incomplete closed traumatic cervical spinal cord injuries. Spine (Phila Pa 1976). 1996;21:2345-51.

2. Koyanagi I, Iwasaki Y, Hida K, Akino M, Imamura H, Abe H. Acute cervical cord injury without fracture or dislocation of the spinal column. J Neurosurg. 2000;93:15-20.

3. Ishida Y, Tominaga T. Predictors of neurologic recovery in acute central cervical cord injury with only upper extremity impairment. Spine (Phila Pa 1976). 2002:27:1652-8. discussion 1658.

4. Penrod LE, Hegde SK, Ditunno Jr JF. Age effect on prognosis for functional recovery in acute, traumatic central cord syndrome. Arch Phys Med Rehabil. 1990;71:963-8.

5. Shepard MJ, Bracken MB. Magnetic resonance imaging and neurological recovery in acute spinal cord injury: observations from the National Acute Spinal Cord Injury Study 3. Spinal Cord. 1999;37:833-7.

6. Choi JH, Shin JJ, Kim TH, Shin HS, Hwang YS, Park SK. Does intramedullary signal intensity on MRI affect the surgical outcomes of patients with ossification of posterior longitudinal ligament? J Korean Neurosurg Soc. 2014;56:121-9.

7. Wada E, Yonenobu K, Suzuki S, Kanazawa A, Ochi T. Can intramedullary signal change on magnetic resonance imaging predict surgical outcome in cervical spondylotic myelopathy? Spine (Phila Pa 1976). 1999;24:455-61. discussion 462.

8. Morio Y, Teshima R, Nagashima H, Nawata K, Yamasaki D, Nanjo Y. Correlation between operative outcomes of cervical compression myelopathy and MRI of the spinal cord. Spine (Phila Pa 1976). 2001;26:123845.

9. Shin JJ, Jin BH, Kim KS, Cho YE, Cho WH. Intramedullary high signal intensity and neurological status as prognostic factors in cervical spondylotic myelopathy. Acta Neurochir (Wien). 2010;152:1687-94.

10. Onishi E, Sakamoto A, Murata S, Matsushita M. Risk factors for acute cervical spinal cord injury associated with ossification of the posterior longitudinal ligament. Spine (Phila Pa 1976). 2012;37:660-6.

11. Gu Y, Chen L, Dong RB, Feng Y, Yang HL, Tang TS. Laminoplasty versus conservative treatment for acute cervical spinal cord injury caused by ossification of the posterior longitudinal ligament after minor trauma. Spine J. 2014;14:344-52.

12. Lucas JT, Ducker TB. Motor classification of spinal cord injuries with mobility, morbidity and recovery indices. Am Surg. 1979;45:151-8.

13. Tsuyama N. Ossification of the posterior longitudinal ligament of the spine. Clin Orthop Relat Res. 1984;71-84.

14. Fujiyoshi T, Yamazaki M, Kawabe J, Endo T, Furuya T, Koda M, et al. A new concept for making decisions regarding the surgical approach for cervical ossification of the posterior longitudinal ligament: the K-line. Spine (Phila Pa 1976). 2008;33:E990-993.

15. Bracken MB, Shepard MJ, Collins WF, Holford TR, Young W, Baskin DS, et al. A randomized, controlled trial of methylprednisolone or naloxone in the treatment of acute spinal-cord injury. Results of the Second National Acute Spinal Cord Injury Study. N Engl J Med. 1990;322:1405-11.

16. Matsunaga S, Sakou T, Hayashi K, Ishidou Y, Hirotsu M, Komiya S. Traumainduced myelopathy in patients with ossification of the posterior longitudinal ligament. J Neurosurg. 2002;97:172-5.

17. Boldin C, Raith J, Fankhauser F, Haunschmid C, Schwantzer G, Schweighofer F. Predicting neurologic recovery in cervical spinal cord injury with postoperative MR imaging. Spine (Phila Pa 1976). 2006;31:554-9.

18. Shin J-J. Intramedullary signal intensity and other prognostic factors for surgical outcome in spinal cord injury associated with ossification of the posterior longitudinal ligament (OPLL). The Spine Journal. 2014;14:S8-9.

19. Ramanauskas WL, Wilner HI, Metes JJ, Lazo A, Kelly JK. MR imaging of compressive myelomalacia. J Comput Assist Tomogr. 1989;13:399-404.

20. Roth EJ, Lawler MH, Yarkony GM. Traumatic central cord syndrome: clinical features and functional outcomes. Arch Phys Med Rehabil. 1990;71:18-23.

21. Schaefer DM, Flanders AE, Osterholm JL, Northrup BE. Prognostic significance of magnetic resonance imaging in the acute phase of cervical spine injury. J Neurosurg. 1992;76:218-23.

22. Kulkarni MV, Bondurant FJ, Rose SL, Narayana PA. 1.5 tesla magnetic resonance imaging of acute spinal trauma. Radiographics. 1988;8:1059-82.

23. Sieh KM, Leung SM, Lam JS, Cheung KY, Fung KY. The use of average Pavlov ratio to predict the risk of post operative upper limb palsy after posterior cervical decompression. J Orthop Surg Res. 2009;4:24.

24. Ramon S, Dominguez R, Ramirez L, Paraira M, Olona M, Castello T, et al. Clinical and magnetic resonance imaging correlation in acute spinal cord injury. Spinal Cord. 1997;35:664-73.

25. Flanders AE, Spettell CM, Friedman DP, Marino RJ, Herbison GJ. The relationship between the functional abilities of patients with cervical spinal cord injury and the severity of damage revealed by MR imaging. AJNR Am J Neuroradiol. 1999;20:926-34.

26. Marciello MA, Flanders AE, Herbison GJ, Schaefer DM, Friedman DP, Lane I. Magnetic resonance imaging related to neurologic outcome in cervical spinal cord injury. Arch Phys Med Rehabil. 1993;74:940-6.

27. Lao L, Zhong G, Li X, Qian L, Liu Z. Laminoplasty versus laminectomy for multi-level cervical spondylotic myelopathy: a systematic review of the literature. J Orthop Surg Res. 2013;8:45.

28. Cho YE, Shin JJ, Kim KS, Chin DK, Kuh SU, Lee JH, et al. The relevance of intramedullary high signal intensity and gadolinium (Gd-DTPA) enhancement to the clinical outcome in cervical compressive myelopathy. Eur Spine J. 2011;20:2267-74.

29. Fujimori T, Iwasaki M, Okuda S, Takenaka S, Kashii M, Kaito T, et al. Longterm results of cervical myelopathy due to ossification of the posterior longitudinal ligament with an occupying ratio of $60 \%$ or more. Spine (Phila Pa 1976). 2014;39:58-67.

30. Nagashima H, Nanjo Y, Tanida A, Mihara T, Takeda C, Teshima R. Influence of spinous process spacers on surgical outcome of laminoplasty for OPLL. Orthopedics. 2013;36:e494-500.

\section{Submit your next manuscript to BioMed Central and take full advantage of:}

- Convenient online submission

- Thorough peer review

- No space constraints or color figure charges

- Immediate publication on acceptance

- Inclusion in PubMed, CAS, Scopus and Google Scholar

- Research which is freely available for redistribution 